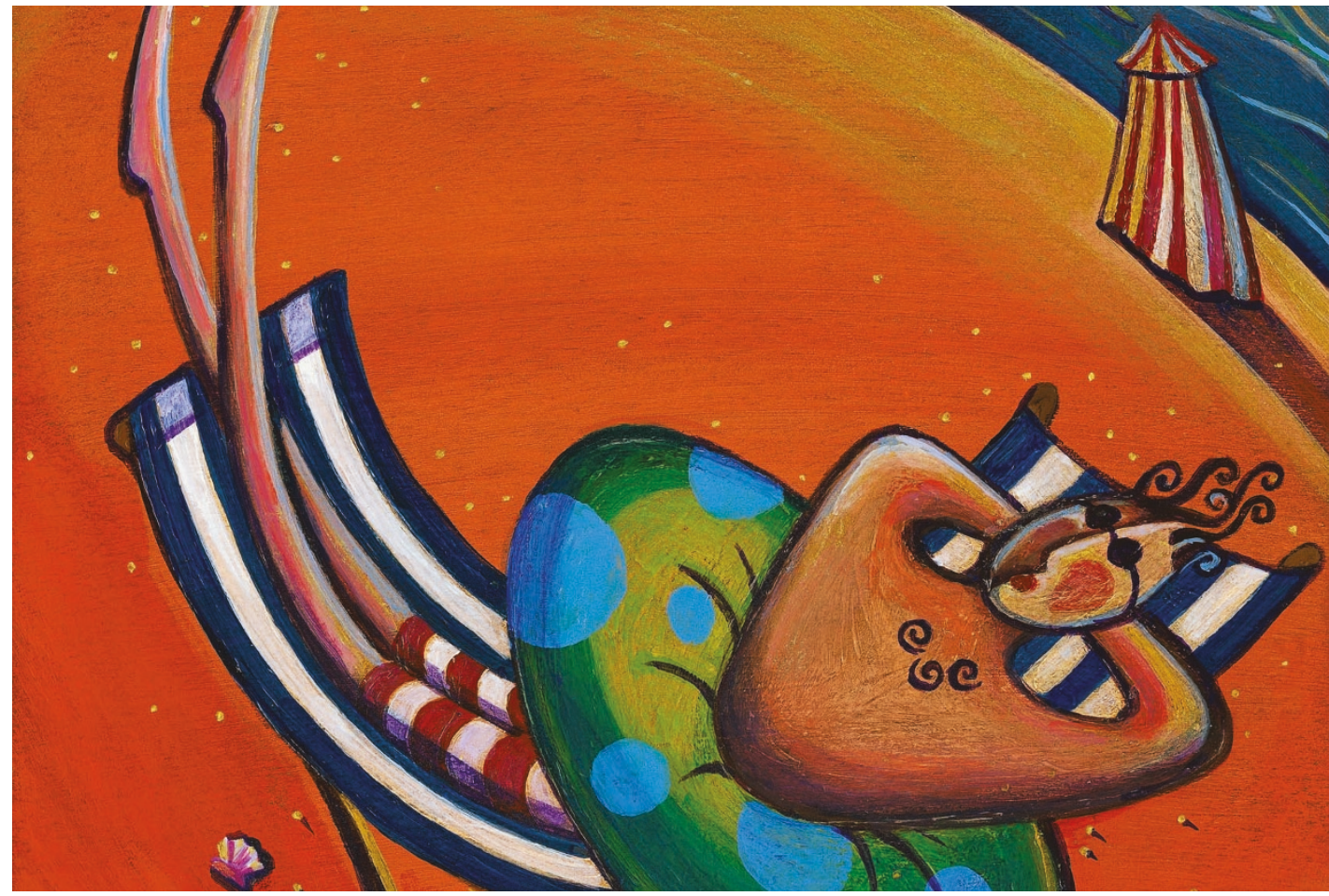

SIGNALLING

\section{Avoiding shade}

(LDs), weakly promotes flowering under LD conditions, whereas phyB, which does not, delays flowering under both SD and LD conditions.

In their study, Cerdán and Chory first identified Arabidopsis seedlings that were defective in phyB signalling, and they scored the defective seedlings for faults in flowering time. This allowed them to identify PFT1 (PHYTOCHROME AND FLOWERING TIME 1), a protein that might function specifically in phy signalling downstream of phyA and phyB.

Next, Cerdán and Chory monitored the flowering time of various mutant plants ( $p f t 1$, phyA and $p h y B$ combinations) under SD and LD conditions. Under both sets of conditions, they found that $p f t 1$ plants were late flowering and that $p f t 1$ could block the early-flowering phenotype of $p h y B$ plants. They also found that $p f t 1$ plants responded strongly to photoperiod. They therefore proposed that "...the main role of PFT1 in phytochrome signalling is to regulate flowering time downstream of phyB in a photoperiod-independent pathway".

When the authors cloned PFT1, they found that it encodes a protein that has features similar to some transcriptional activators. Furthermore, by tagging PFT1 with green fluorescent protein, they showed that it is present in the nucleus. In addition, they found that phyB probably regulates PFT1 post-transcriptionally, because the phyB mutation barely affects PFT1 messenger RNA levels.

So, how does $p f t 1$ block the phyB early-flowering phenotype?
FT integrates several flowering-time pathways, so Cerdán and Chory analysed $F T$ mRNA levels in wildtype, $p f t 1$, phyB and $p f t 1$ phyB plants. They found that FT mRNA levels are higher in $p h y B$ than in wild-type plants, and that $F T$ mRNA levels were low in the $p f t 1$ and $p f t 1 p h y B$ plants. These results indicate that phyB could have an inhibitory effect on PFT1, which would otherwise activate its downstream target FT.

The authors confirmed that the phyB-PFT1-FT pathway functions independently of the photoperiod pathway by showing that there is no significant correlation between $C O$ mRNA levels and the flowering time of $p f t 1$ or $p h y B$ plants. In addition, they verified that PFT1 has a specific role in a phyB pathway that regulates flowering time in response to light-quality changes by showing that $p f t 1$ plants are unable to accelerate flowering in response to shade conditions.

Cerdán and Chory therefore propose that the phyB-PFT1-FT pathway is a photoperiod-independent lightquality sensing pathway that triggers flowering in response to a low ratio of red to far-red incoming light. This work has clarified this role of phyB in shade avoidance, and, if plants could be made to delay flowering even when they are shaded by their neighbours, it might also have implications for increasing crop yields.

Rachel Smallridge which induces flowering. phyA and phyB regulate flowering in opposite ways - phyA, which discriminates short days (SDs) from long days
H I G H L I G H T S

\section{IN THE NEWS}

No longer just an empty sack Bacteria possess organelles - acidic calcium storage compartments, known as acidocalcisomes - that are also present in eukaryotes, according to a recent report in The Journal of Biological Chemistry.

For years, students have been taught that bacteria are 'empty sacks'. But now, research by Roberto Docampo and his colleagues from the University of Illinois, Urbana, indicates that bacteria have specialized compartments that are equivalent to eukaryotic organelles.

\section{Bacteria contain} subcellular entities, known as volutin granules, which were previously thought to lack an enclosing membrane and enzymatic activity. However, volutin granules (or acidocalcisomes) of unicellular eukaryotes are surrounded by a membrane that contains enzymatic pumps and exchangers.

Choosing Agrobacterium tumefaciens - which contains a proton pyrophosphatase ( $\mathrm{H}^{+}$-PPase), a marker for acidocalcisomes - for his studies, Docampo investigated whether the bacterial volutin granules have characteristics similar to the acidocalcisomes.

The researchers found that each bacterial granule was surrounded by a membrane. Then, using antibodies against A. tumefaciens $\mathrm{H}^{+}$-PPase, they showed the presence of an $\mathrm{H}^{+}$-PPase in the membrane.

The origin of the organelles is unknown. Usually, organelles are either components of an intracellular trafficking system, or they evolved from formerly free-living organisms. "Which of these might happen in bacteria is not clear", says Andrew Roger from Dalhousie University in Nova Scotia (Nature, 26 June 2003). Arianne Heinrichs 